\title{
On the Connected End Equitable Domination of Graphs
}

\author{
Murthy K.B \\ Department of Agril.Statistics \& Applied Mathematics \\ University of Agricultural Sciences, GKVK Campus, \\ Bengaluru, India. \\ kbmurthy2005@gmail.com
}

\begin{abstract}
A$ set $S$ of vertices in a graph $G$ is a connected dominating set if every vertex not in $S$ is adjacent to some vertex in $S$ and the subgraph induced by $S$ is connected. The connected domination number $\gamma_{c}(G)$ is the minimum size of such a set. An equitable dominating set $D$ in a graph $G$ is called connected equitable dominating set in the subgraph $\langle D>$ induced by $D$ is connected. In this paper we introduce the connected end equitable domination of graphs. The values of this concept are found for some important families of graphs and some relations with some other domination parameters are presented.
\end{abstract}

Keywords: connected end equitable domination, helm graph, tadpole graph (dragon graph).

AMS Mathematics Subject Classification (2000):05C50.

\section{INTRODUCTION}

The connected domination of a graph has been introduced by Sampathkumar and Walikar [4].

A dominating set $S$ is said to be connected dominating set if the induced subgraph $\langle S\rangle$ is connected. The minimum of the cardinalities of the connecting dominating set is called connected domination of $G$ and denoted by $\gamma_{c}(G)$. The connected equitable domination number has been introduced in [4] and [5] independently. An equitable dominating set $D$ in a connected graph $G$ is called connected equitable dominating set in the subgraph $\langle D\rangle$ induced by $D$ is connected in $G$. The minimum cardinality of a connected equitable dominating set of $G$ is called equitable domination number of $G$ and denoted by $\gamma_{c e}(G)$.

In general the connected dominating set has a lot of application in network see [6] that motivated us in this section to define the connected end equitable dominating set and the connected end equitable domination number of graphs. Also before defining the connected end equitable domination number in a graph we define the connected end domination in a graph.

\section{Connected End Equitable Domination Number}

Definition 2.1. Let $G=(V, E)$ be a connected graph. An end dominating set $D \subseteq V(G)$ is called connected end dominating set if the induced subgraph $\langle D\rangle$ is connected. Any connected end dominating set with minimum cardinality is called minimum connected end dominating set. The connected end domination number is the cardinality of a minimum connected dominating set and denoted by $\gamma^{\text {ce }}(G)$.

Definition 2.2. Let $G=(V, E)$ be connected graph. An end equitable dominating set $D$ is called connected end equitable dominating set if the subgraph $\langle D\rangle$ which is induced by $D$ in $G$ isconnected. The minimum cardinality of a connected end equitable dominating set is called the connected end equitable domination number of $G$ and denoted by $\gamma_{c e e}(G)$. 
Example 2.3. Let $G$ be a connected graph in Figure 1

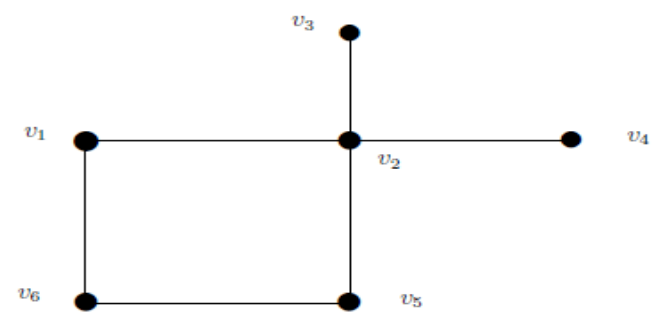

Fig. $1: \gamma_{\text {cee }}(G)=5$

From the Figure 2.1, it is easy to see that the set $\left\{v_{2}, v_{6}\right\}$ is a minimum dominating set or $\left\{v_{2}, v_{5}\right\} \operatorname{or}\left\{v_{2}, v_{1}\right\}$ and hence $\gamma(G)=2$ and $\left\{v_{1}, v_{2}\right\}$ is a minimum connected dominating set that means $\gamma_{c}(G)=2$.

The set $\left\{v_{3}, v_{4}, v_{6}\right\}$ is one of the minimum end dominating sets of $G$. Therefore $\gamma^{e}(G)=3$ and the minimum connected end dominating sets are $\left\{v_{3}, v_{4}, v_{2}, v_{5}\right\}$ or $\left\{v_{3}, v_{4}, v_{2}, v_{1}\right\}$.

Therefore, $\gamma^{c e}(G)=4$.

The minimum equitable dominating sets are $\left\{v_{2}, v_{3}, v_{4}, v_{6}\right\}$ and also $\left\{v_{2}, v_{3}, v_{4}, v_{6}\right\}$ is end equitable dominating set . Therefore, $\gamma_{e}(G)=\gamma_{e c}(G)=4$.

There are two minimum end equitable dominating sets in $G\left\{v_{2}, v_{3}, v_{4}, v_{5}, v_{6}\right\}$ and $\left\{v_{2}, v_{3}, v_{4}, v_{1}, v_{6}\right\}$.

Hence, $\gamma_{c e e}(G)=5$.

Observation 2.4. For any connected graph $G=(V, E)$ the connected end equitable dominating set is defined.

Proof: The proof is straightforward by taking $D=V(G)$ then $D$ is connected end equitable dominating set.

Proposition 2.5. For any connected graph $G=(V, E)$

$$
\text { We have } \quad \gamma(G) \leq \gamma_{e}(G) \leq \gamma_{e e}(G) \leq \gamma_{c e e}(G) .
$$

Proof: Let $G$ be any connected graph and let $D_{1} \subseteq V(G)$ is any minimum connected end equitable dominating set in $G$. Then clearly $D_{1}$ is also an end equitable dominating set in $G$. Therefore, $\gamma_{e e}(G) \leq$ $\left|D_{1}\right|=\gamma_{c e e}(G)$.

Suppose that $D_{2}$ is any minimum end equitable dominating set of $G$. Then $D_{2}$ is also an equitable dominating set by the definition of the end equitable dominating set.

Therefore, $\gamma_{e}(G) \leq\left|D_{2}\right|$.

Hence, $\gamma_{e}(G) \leq \gamma_{e e}(G)$. Similarly any equitable dominating set is dominating set we have,

$$
\gamma(G) \leq \gamma_{e}(G) .
$$

Hence, $\gamma(G) \leq \gamma_{e}(G) \leq \gamma_{e e}(G) \leq \gamma_{c e e}(G)$.

Remark 2.6. The equality in proposition 2.5 can be hold in many graphs which has no end vertices for example

$$
\begin{aligned}
\gamma\left(C_{4}\right)=\gamma_{e}\left(C_{4}\right)=\gamma_{e e}\left(C_{4}\right) & =\gamma_{c e e}\left(C_{4}\right)=4 \\
\gamma\left(k_{p}\right) & =\gamma_{e}\left(k_{p}\right)=\gamma_{e e}\left(k_{p}\right)=\gamma_{c e e}\left(k_{p}\right)=1
\end{aligned}
$$

Proposition 2.7. Let $G$ be a connected graph. Then, $\gamma_{c}(G) \leq \gamma_{c e e}(G)$.

Proof: Let $D \subseteq V(G)$ be minimum connected equitable dominating set of the connected graph $G$.Then $D$ is dominating set of $G$ and the induced subgraph $\langle D\rangle$ is connected . Therefore, $D$ is also 
connected dominating set i.e., any connecting end equitable dominating set is also connected dominating set.

Hence, $\gamma_{c}(G) \leq \gamma_{c e e}(G)$.

Corollary 2.8. For any $k$-regular or $(k, k+1)$ bi-regular graph $\gamma_{c}(G)=\gamma_{c e e}(G)$.

Proof: We have $\gamma_{c}(G) \leq \gamma_{c e e}(G)$ by Proposition 2.7, now let $D$ be minimum connected dominating set of connected $k$-regular or $(k, k+1)$ bi-regular graph.

By the definition of equitable dominating set it is easy to see that $D$ is also equitable dominating set and also $\langle D\rangle$ is connected and since $G$ is $k$-regular or $(k, k+1)$ bi-regular that means $D$ is also connected end equitable dominating set.

Proposition 2.9. Let $G$ be a connected graph. Then, $\gamma_{e e}(G)=\gamma_{c e e}(G)=1$ if and only if $G$ has no end vertex and there is at least one vertex $v \in V(G)$ such that $\operatorname{deg}_{e}(v)=n-1$ where $n$ is the number of vertices in $G$.

Proof: Let $G$ be a connected graph has no end vertex i.e., $\delta(G) \geq 2$ and there exists one vertex say $u \in V(G)$ such that $\operatorname{deg}_{e}(u)=n-1$ where $n$ is the number of vertices in $G$. Then the set $\{u\}$ is connected end equitable dominating set of $G$ and by Proposition 2.5 , we get $\gamma_{e e}(G)=\gamma_{c e e}(G)=1$.

Conversely, suppose $G$ is connected graph and $\gamma_{e c}(G)=\gamma_{c e e}(G)=1$, then $G$ has no end vertex and there is $D=\{w\}$ which is connected end equitable dominating set .

Therefore, $\operatorname{deg}_{e}(w)=n-1$.

Theorem 2.10. Let $G=(V, E)$ be any tree with $n$ vertices or $G$ be totally equitable disconnected with $n$ vertices.Then, $\gamma_{c e e}(G)=n$

Proof: Let $G=(V, E)$ such that $G$ is tree of $n$ vertices, if we take $D \in V(G)$ then $D$ is connected end equitable dominating set. Therefore, $\gamma_{c e e}(G) \leq n$.

Now let $\gamma_{c e e}(G) \leq n-1$ then there exists at least one vertex $v \in V(G)$ does not belong to the minimum end equitable dominating set that is $D=V-\{w\}$ is connected end equitable dominating set and $\operatorname{deg}(w) \geq 2$ then if $\langle V-\{w\}\rangle$ is connected implies that $G$ has a cycle that is contradiction that $G$ is a tree (since every vertex other than the end vertex in a tree is cut vertex).Hence there is no connected end equitable dominating set of size less than -1 .

Hence, $\gamma_{c e e}(G)=n$.

Similarly, if $G$ is equitable totally disconnected then $\gamma_{c e e}(G)=n$.

Corollary 2.11. For any path $P_{n}$, with $n \geq 2$ vertices, $\gamma_{\text {cee }}\left(P_{n}\right)=n$.

Proposition 2.12. For any cycle $C_{n}$ on $n \geq 3$ vertices, $\gamma_{\text {cee }}\left(C_{n}\right)=n-2$.

Proof: Since $\gamma_{c}\left(C_{n}\right)=n-2$ and let $D=V-\{v, u\}$ is any subset of the vertices on the cycle $C_{n}$ such that $u$ and $v$ any adjacent vertices. Clearly $D$ is connected end equitable dominating set of $C_{n}$ that means $\gamma_{c e e}(G) \leq n-2$ and by proposition 2.7, we have $n-2=\gamma_{c}\left(C_{n}\right) \leq \gamma_{c e e}\left(C_{n}\right)$.

Hence, $\gamma_{c e e}\left(C_{n}\right)=n-2$.

Corollary 2.13. For any bi-star graph $B(m, n), \gamma_{c e e} B(m, n)=m+n+2$

Corollary 2.14. For any spider graph $G ¥ S P_{n}, \gamma_{c e e}(G)=2 n+1$.

Proposition 2.15. For any complete bipartite graph $G \cong k_{m, n}$,

$$
\gamma_{e c}(G)=\gamma_{c e e}(G)=\left\{\begin{array}{lr}
m+n & |m+n| \geq 2 ; \\
2 & \text { otherwise }
\end{array}\right.
$$

Proof:

Case1. If $G \cong k_{m, n}$ and $|m+n| \geq 2$, in this case the graph $G$ is equitable totally disconnected and By the Proposition 2.10, $\gamma_{e e}(G)=m+n$ and by the Proposition $2.5 \gamma_{e c}(G) \leq \gamma_{c e e}(G)$. 
Hence, $\gamma_{e c}(G)=\gamma_{c e e}(G)=m+n$.

Case2. If $G \cong k_{m, n}$, where $|m+n| \leq 1$, then if $A, B$ the partite sets of $G$,by selecting one vertex $v \in A$ and $u \in B$, then $D=\{u, v\}$ is connected equitable end dominating set

$\gamma_{e e}=\gamma_{c e e}(G) \leq 2$. But by the Proposition $2.9, \gamma_{c e e}(G) \neq 1$.

Hence, $\gamma_{e c}(G)=\gamma_{c e e}(G)=2$.

Observation 2.16. For any connected graph $G$ any minimum connected equitable dominating set must be contained all the supporting vertices.

Proof: Let $G$ be any connected graph and let $D \subseteq V(G)$ be any connected equitable end dominating set. Suppose that $u$ be any end vertex in $G$ and $v$ is its supporting vertex and suppose that $v \notin D$ then $\langle D\rangle$ is not connected because the two vertices $u, v$ has no path connected to them.

Hence, $D$ must contain all the supporting vertices.

Proposition2.17. Let Gbe any connected graph with $t$ end vertices $n \geq 3$ vertices.

$$
\text { Then, } \gamma_{c e e}(G) \geq t+1 \text {. }
$$

Proof: Clearly if $G$ is connected graph with $n \leq 2$ vertices then we have two cases either $G \cong k_{1}$ or $G \cong k_{2}$ and in both cases $\gamma_{c e e}(G)$ is equal to the number of vertices.

If $n \geq 3$, then we have two cases.

Case 1: If $G$ has no end vertices, then it is obvious that in general $\gamma_{c e e}(G) \geq 1$ that means

$$
\gamma_{c e e}(G) \geq t+1 \text { as } t=0 .
$$

Case 2: If $G$ has $t \geq 1$ end vertices. Let $D$ be any connected equitable end dominating set of $G$, then the $t$ end vertices and their supporting vertices must belong to the dominating set. So the question is to how much can minimize the supporting vertices. The answer is obviously if $s$ is denoted the number of supporting vertices in $G$ then $1 \leq s \leq t$. That means we can minimize the number of supporting vertices till one vertex.

Hence, any connected equitable end dominating set contains at least $t$ end vertices and one supporting vertex. Hence, $\gamma_{c e e}(G) \leq t+1$.

Further the bound in Proposition 2.17 is sharp if $G \cong k_{1, n}, n \geq 2$.

Proposition 2.18. For any connected graph $G$ with $t \geq 1$ end vertices.

$$
\gamma_{\text {cee }}(G)=t \text { if and only if } G \cong k_{2} \text {. }
$$

(ii) let $H$ be connected graph with $m \geq 3$ vertices and $G \cong H \circ k_{1}$.

Then, $\gamma_{c e e}(G)=2 t=2 m$, where $t$ is the number of end vertices.

Proof: (i) If $G \cong k_{2}$ then $t=2$ and clearly, $\gamma_{c e e}\left(k_{2}\right)=2$.

Conversely, suppose $\gamma_{c e e}(G)=t$, where $t \geq 1$ as we seen in Proposition 2.17 if the number of vertices of the graph $n \geq 3$, the number of supporting vertices at least equal to one that means $G$ must contain $n \leq 2$ vertices .

Hence $\gamma_{\text {cee }}(G)=t$.

(ii) Let $G \cong H \circ k_{1}$. If $H$ has $m \geq 3$ vertices then clearly there are $t=m$ end vertices and all the other vertices of $H$ will be pendent vertices. Hence any connected equitable end dominating set of $H \circ k_{1}$ is contained $2 t$ vertices.

Remark 2.19. The converse of (ii) in the Proposition 2.18 is not correct. See the following example 2.20 
Example 2.20. Let $G$ be connected graph as in Figure.2.

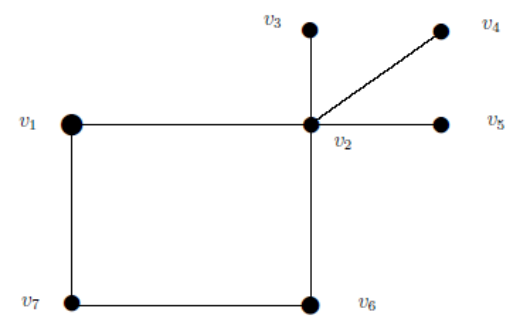

Fig. 2: The two minimum connected equitable end dominating set in $G$

Then clearly, $\left\{v_{2}, v_{3}, v_{4}, v_{5}, v_{6}, v_{7}\right\}$ and $\left\{v_{2}, v_{3}, v_{4}, v_{5}, v_{1}\right\}$ are only the two minimum connected equitable end dominating set .That means, $\gamma_{c e e}(G)=6=3 t$, where $t=3$, the number of end vertices but $G ¥ H \circ k_{1}$.

Proposition2.21. For any positive integer $a \geq 1$ there exists a graph $G$ such that $\gamma_{\text {cee }}(G)=2 a$.

Proof: By taking $H$ is the path $P_{a}$ and $G \cong P_{a} \circ k_{1}$. Then clearly, $\gamma_{c e e}(G)=2 a$.

Proposition2.22. Let $G$ be a cycle with at least one edge attach to each vertex in the cycle.

Then,$\gamma_{c e e}(G)=n$, where $n$ is the number of vertices in $G$.

Proof: Let $G$ be a cycle with at least one edge attach to each vertex in the cycle.

Suppose there is a connected equitable end dominating set $D$ which has $m$ vertices such that $m<n$. Suppose $v$ be any vertex in $V(G)$ such that $v \notin D$, so we have two cases either $v$ is one vertex in the cycle or $v$ is end vertex.

Case 1. If $v$ is one vertex in the cycle, then there exists attached edge say $v u$ where $u$ is end vertex that means $u$ is isolated vertex in the induced subgraph $\langle D\rangle$ which is a contradiction.

Case 2. $v$ is an end vertex which is contradiction since any connected equitable end dominating set must contain all the end vertices.

Example 2.23. Let $G$ be a cycle $C_{5}$ with three attached edges in each vertex as in Figure 3.

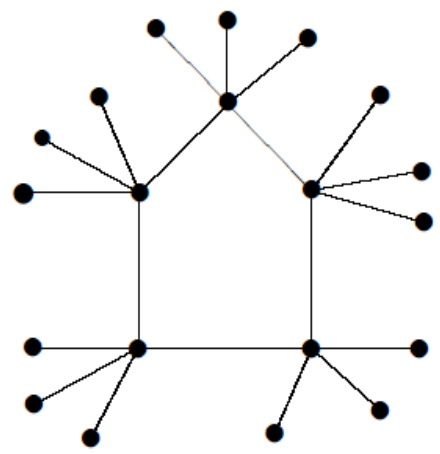

It is easy to see that

Fig. 3: $C_{5} \circ \overline{k_{3}}$

$\left.\gamma^{e} G\right)=15, \quad \gamma(G)=5$

$\gamma_{c}(G)=5, \quad \gamma_{e}(G)=17$

$\gamma_{c e e}(G)=20$ and $\gamma_{e e}(G)=15$.

Corollary 2.24. Let $G \cong C_{m} \circ \overline{k_{p}}$. Then, $\gamma_{c e e}(G)=p m+m$

Proof: It is straightforward by applying Proposition 2.22 and instead to attach one edge in every vertex of the cycle will attach edges in each vertex.

We can generalize the result of Proposition 2.22 as the following result. 
Proposition 2.25. For any connected graph $H$ with $m$ vertices if $G \cong H \circ \overline{k_{p}}$,

then $\gamma_{c e e}(G)=p m+m$.

Proof: Let $D$ be any minimum connected equitable end dominating set of , then all the $m p$ end vertices must belong to $D$ and all the other vertices in $G$ are support vertices and must belong to $D$ to make $\langle D\rangle$ connected .

Hence, $\gamma_{c e e}(G) \geq m p+m$.

Therefore, $\gamma_{c e e}(G)=m p+m$.

The helm graph $H_{n}$ is the graph obtained from the wheel $W_{n}$ of $n+1$ vertices by adjoining a pendent edge at each vertex of the cycle.

See the following helm graphs in Figure 4.
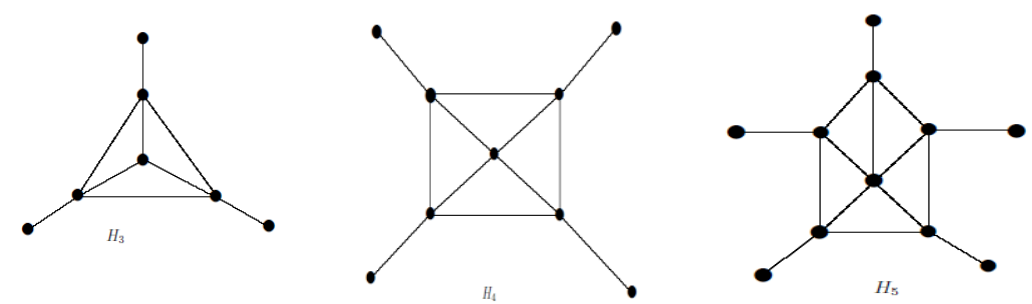

Fig. 4: helm graph

Proposition2.26. Let $G \cong H_{n}$ be helm graph of $2 n+1$ vertices .Then

$$
\gamma_{c e e}(G)=\left\{\begin{array}{c}
2 n \text { if } n=3 \text { or } 4 \\
2 n+1 \text { otherwise. }
\end{array}\right.
$$

Proof:

Case 1 . If $n=3$ or $n=4$ then any minimum connected equitable end dominating set must contain the end vertices and the support $2 n$ vertices remaining the center but the center has degree 3 or 4 then it will dominate by any support vertex.

Case 2. The center has degree more than or equal to 5, that means the center vertex is an equitable isolated vertex and must belong to any connected equitable end dominating set. Similarly the end vertices and the support vertices must belong to any minimum connected equitable end dominating set.

Hence,$\gamma_{c e e}(G)=2 n+1$.

Proposition 2.27. For any tadpole graph $T_{m, n}, \quad \gamma_{c e e}\left(T_{m, n}\right)=m+n-2$

Proof: Let $G \cong T_{m, n}$ be any tadpole graph with $m+n$ vertices as in Figure 5
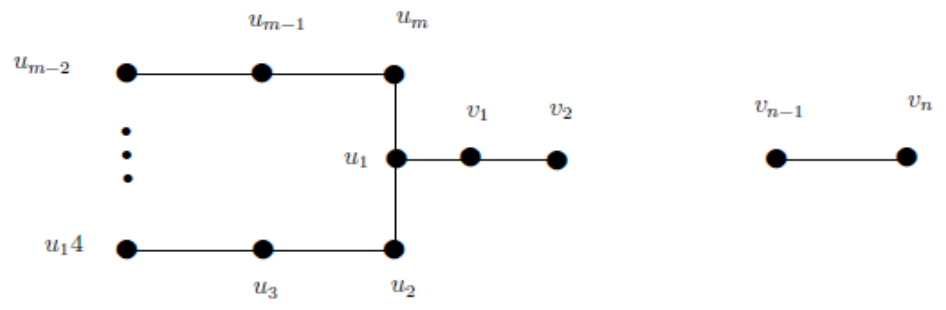

Fig.5: tadpole graph with $m+n$ vertices.

Clearly any connected equitable end dominating set $D$ must contains all the vertices $v_{i}$, where $i=1,2, \ldots \ldots \ldots, n$ since if any vertex $v_{i}$ for any $i$ does not belong to $D$ then $\langle D\rangle$ is not connected and hence $D$ is not connected equitable end dominating set.

Therefore, $\gamma_{c e e}\left(T_{m, n}\right)=n+\gamma_{c e e}\left(C_{m}\right)$ and we have, $\gamma_{c e e}\left(C_{m}\right)=m-2$

Hence, $\gamma_{c e e}\left(T_{m, n}\right)=m+n-2$. 


\section{CONCluSion}

We continue the study of end equitable domination of graphs by defining two types of end equitable domination. We introduce the connected end equitable domination of graphs. The values of this concept are found for some important families of graphs and some relations with some other domination parameters are present.

\section{REFERENCES}

[1] A. Al-Kenani, N. Soner and A. Alwardi, "Graphs and Degree Equitability," Applied Mathematics, Vol. 4 No. 8, 2013, pp. 1199-1203.

[2] Ding-Zhu Du and PanosM.Pardalos (Eds. ), Hand book of Combinatorial Optimization (Vol.5), Kluwer Academic Publishers(2004),329-369.

[3] Harary, F., Graph Theory, Addison Wesley, Massachusetts, 1969.

[4] L. Arseneau, A. Finbow, B. Hartnell, A. Hynick, D. Maclean and L. O'sullivan, On minimal connected dominating sets, JCMCC 24 (1997), 185-191.

[5] Sampathkumar,E. \& Walikar,H.B., The connected domination number of a graph, J.Math.Phys.Sci. 13 (1979), 607-613.

[6] Sampathkumar, E., On some new domination parameters of a graph, A Survey Proceedings of a Symposium on Graph Theory Combinatorics, Kochi, Kerala,India, 17-19 May (1991), 7-13.

[7] Sivakumar, S., Soner, N. D \& Anwar Alwardi, Neighborhood connected equitable edge domination in graphs, Research Journal of Pure Algebra,3(3)(2013), 119-125.

[8] S.T. Hedetniemi and R. Laskar, Connected domination in graphs, In: Graph Theory and Combinatorics, B. Bollob’as ed. Academic Press, London 1984, pp. 209-218.

\section{AUTHOR's BIOGRAPHY}

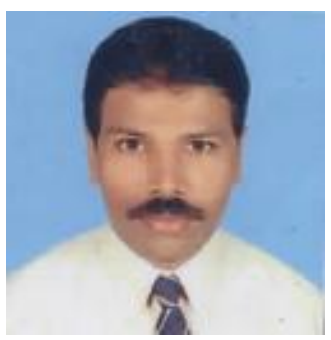

Murthy K.B, is working as an Associate Professor of Mathematics in the Department of Agricultural Statistics and Applied Mathematics, University of Agricultural Sciences, Bengaluru which is a prestigious University of Karnataka State. He has been teaching various courses to undergraduate and postgraduate students for 21 years. He is an active researcher in Graph Theory where he has several publications in international peer- reviewed journals. $\mathrm{He}$ has participated in some National and International seminars and presented his papers on various topics. He has zeal to invent new findings in Graph Theory. 